\title{
The nonlinear vibrations of soil-pile systems under seismic ground motion
}

\author{
E. J. Sapountzakis \& A. E. Kampitsis \\ School of Civil Engineering, \\ National Technical University of Athens, Greece
}

\begin{abstract}
In this paper, a boundary element method (BEM) is developed for the nonlinear dynamic analysis of soil-pile systems accounting for both kinematic and inertial interaction. The column-pile of arbitrary doubly symmetric simply or multiply connected constant cross section is partially embedded in layered viscoelastic profile, undergoing moderate large deflections taking into account the effects of shear deformation and rotary inertia. The column-pile is subjected to seismic action as well as to arbitrarily distributed or concentrated transverse, axial and bending loading. To account for shear deformations, the concept of shear deformation coefficients is used. Five boundary value problems are formulated with respect to the transverse displacements, to the axial displacement and to two stress functions and solved using the Analog Equation Method, a BEM based method. The application of the boundary element technique yields a nonlinear coupled system of equations of motion. The evaluation of the shear deformation coefficients is accomplished from the aforementioned stress functions using only boundary integration. The proposed model takes into account the coupling effects of bending-shear deformations along the member as well as the shear forces along the span induced by the applied axial loading. Numerical examples are solved to illustrate the efficiency and the range of applicability of the developed method.
\end{abstract}

Keywords: soil-pile interaction, nonlinear vibrations, seismic ground motion, large deflections, Timoshenko theory, viscoelastic foundation, boundary element method. 


\section{Introduction}

Many problems related to soil-structure interaction can be modelled as a beam or a beam-column on an elastic foundation. Practical examples of these are railroad tracks, highway pavements, continuously supported pipelines and strip foundations. Moreover, piles are frequently employed for the foundation of structures such as buildings, quay walls, bridges and offshore structures. These piles, which are subjected to lateral forces that result from loading on supported structures, develop a nonlinear dynamic response to an earthquake excitation. Thus, the study of nonlinear effects on the dynamic analysis of structural elements is essential in civil engineering applications, wherein weight saving is of paramount importance. This non-linearity results from retaining the square of the slope in the strain-displacement relations (intermediate non-linear theory), avoiding in this way the inaccuracies arising from a linearized second-order analysis. Thus, the aforementioned study takes into account the influence of the action of axial, lateral forces and end moments on the deformed shape of the structural element. Moreover, due to the intensive use of materials having relatively high transverse shear modulus and the need for beam members with high natural frequencies the error incurred from the ignorance of the effect of shear deformation may be substantial, particularly in the case of heavy lateral loading. The Timoshenko-Rayleigh beam theory, which includes shear deformation and rotary inertia effects has an extended range of applications as it allows treatment of deep beam (depth is large relative to length), short and thin-webbed beams and beams where higher modes are excited.

When the beam-column deflections of the structure are small, a wide range of linear analysis tools, such as modal analysis, can be used and some analytical results are possible. During the past few years, the linear dynamic analysis of beams on elastic foundation has received a lot of attention in the literature with the pioneer work of Hetenyi [1] who studied the elementary Bernoulli-Euler beams on elastic Winkler foundation. Rades [2] presented the steady-state response of a finite rigid beam resting on a foundation defined by one inertial and three elastic parameters with the assumption of a permanent and smooth contact between beam and foundation considering only uncoupled modes. Wang and Stephens [3] studied the natural vibrations of a Timoshenko beam on a Pasternak-type foundation showing the effects of rotary inertia, shear deformation and foundation constants of the beam employing general analytic solutions for simple cases of boundary conditions. De Rosa [4] and El-Mously [5] derived explicit formulae for the fundamental natural frequencies of finite Timoshenko-beams mounted on finite Pasternak foundation.

Further research by El Naggar and Novak [6] was concerned with the lateral response of single piles and pile groups accounting the nonlinear behaviour of the soil adjacent to the pile and discontinuity conditions at the pile-soil interface. Padron et al. [7] studied a BEM-FEM coupling model for the time harmonic dynamic analysis of piles and pile groups embedded in an elastic half-space where piles are modelled using finite beam elements according to the Bernoulli hypothesis, while the soil is modelled as semi-infinite, isotropic, homogeneous or 
zoned homogeneous, linear, viscoelastic medium using boundary elements. Hu et al. [8] presented the nonlinear partial differential equation governing the nonlinear transverse vibration of pile under the assumption that the materials of both the pile and the soil obey nonlinear elastic and linear viscoelastic constitutive relations while the frequency and the response of the system have been obtained by the complex mode method and the method of multiple time scales.

As the deflections become larger, the induced geometric nonlinearities result in effects that are not observed in linear systems. Contrary to the large amount of attention in the literature given to the linear dynamic analysis of beam-columns supported on elastic foundation, little work has been done on the corresponding nonlinear problem, such as the nonlinear free vibration analysis of multispan beams on elastic supports presented by Lewandowski [9], employing the dynamic finite element method, neglecting the horizontally and rotary inertia forces and considering the beams as distributed mass systems.

In this paper, a boundary element method is developed for the nonlinear dynamic analysis of soil-pile systems accounting for both kinematic and inertial interaction. The column-pile of arbitrary, doubly symmetric simply or multiply connected, constant cross section is partially embedded in layered viscoelastic profile, undergoing moderately large deflections taking into account the effects of shear deformation and rotary inertia. The column-pile is subjected to seismic action as well as to arbitrarily distributed or concentrated transverse, axial and bending loading. To account for shear deformations, the concept of shear deformation coefficients is used. Five boundary value problems are formulated with respect to the transverse displacements, to the axial displacement and to two stress functions and solved using the Analog Equation Method [10], a BEM based method. The application of the boundary element technique yields a nonlinear coupled system of equations of motion. The solution of this system is accomplished iteratively by employing the Average Acceleration Method in combination with the Modified Newton Raphson Method [11, 12]. The evaluation of the shear deformation coefficients is accomplished from the aforementioned stress functions using only boundary integration. The proposed model takes into account the coupling effects of bending-shear deformations along the member as well as the shear forces along the span induced by the applied axial loading. Numerical examples are worked out to illustrate the efficiency and the range of applications of the developed method. The essential features and novel aspects of the present formulation compared with previous ones are summarized as follows:

i. The proposed method is capable of taking into account both kinematic and inertial interaction to the geometrical nonlinear dynamic response of piles.

ii. The site seismic response is obtained through one dimensional shear wave propagation analysis.

iii. The layered linear half-space is approximated as a viscoelastic foundation.

iv. The pile head and tip are supported by the most general nonlinear boundary conditions.

v. Shear deformation effect and rotary inertia are taken into account. 
vi. The proposed model takes into account the coupling effects of bendingshear deformations along the member as well as shear forces along the span induced by the applied axial loading.

vii. The shear deformation coefficients are evaluated using an energy approach, instead of Timoshenko and Goodier's [13] and Cowper's [14].

viii. The proposed method employs a BEM approach (requiring boundary discretization) resulting in line or parabolic elements instead of the area elements of the FEM solutions (requiring the whole cross section to be discretized into triangular or quadrilateral area elements), while a small number of line elements are required to achieve high accuracy.

\section{Statement of the problem}

A prismatic column-pile of length $l$ and constant, arbitrary doubly symmetric cross-section of area $A$ is considered. The homogeneous isotropic and linearly elastic material of the column-pile cross-section, with modulus of elasticity $E$, shear modulus $G$ and Poisson's ratio $v$ occupies the two dimensional multiply connected region $\Omega$ in the $y$-z plane, bounded by the $\Gamma_{j}(j=1,2, \ldots, K)$ boundary curves, which are piecewise smooth, i.e. they may have a finite number of corners; $C y z$ is the principal bending coordinate system through the cross section's centroid. The column-pile is partially embedded in a layered soil profile. The foundation model is characterized by the Winkler moduli $k_{y}, k_{z}$ and the damping coefficients $c_{y}, c_{z}$, in the directions $\mathrm{y}, z$, respectively. Thus, the foundation reaction is written as

$$
\begin{aligned}
& p_{s y}(x, t)=k_{y} v(x, t)+c_{y} \frac{\partial v(x, t)}{\partial t} \\
& p_{s z}(x, t)=k_{z} w(x, t)+c_{z} \frac{\partial w(x, t)}{\partial t}
\end{aligned}
$$

The column-pile is subjected to seismic action as well as to the combined action of the arbitrarily distributed or concentrated time dependent axial loading $p_{x}=p_{x}(x, t)$, transverse loading $p_{y}=p_{y}(x, t), p_{z}=p_{z}(x, t)$ acting in the $y, z$ directions, respectively, and bending moments $m_{y}=m_{y}(x, t), m_{z}=m_{z}(x, t)$ about $y, z$ axes, respectively.

Under the action of the aforementioned loading, the displacement field of the column-pile, taking into account shear deformation effect, is given as

$$
\begin{aligned}
& \bar{u}(x, y, z, t)=u(x, t)-y \theta_{z}(x, t)+z \theta_{y}(x, t) \\
& \bar{v}(x, t)=v(x, t) \quad \bar{w}(x, t)=w(x, t)
\end{aligned}
$$


where $\bar{u}, \bar{v}, \bar{w}$ are the axial and transverse column-pile displacement components with respect to the $C y z$ system of axes; $u(x, t), v(x, t), w(x, t)$ are the corresponding components of the centroid $C$ and $\theta_{y}(x, t), \theta_{z}(x, t)$ are the angles of rotation due to bending of the cross-section with respect to its centroid.

Employing the strain-displacement relations of three-dimensional elasticity for moderate displacements, the following strain components can be easily obtained

$$
\begin{gathered}
\varepsilon_{x x}=\frac{\partial \bar{u}}{\partial x}+\frac{1}{2}\left[\left(\frac{\partial \bar{v}}{\partial x}\right)^{2}+\left(\frac{\partial \bar{w}}{\partial x}\right)^{2}\right] \\
\gamma_{x z}=\frac{\partial \bar{w}}{\partial x}+\frac{\partial \bar{u}}{\partial z}+\left(\frac{\partial \bar{v}}{\partial x} \frac{\partial \bar{v}}{\partial z}+\frac{\partial \bar{w}}{\partial x} \frac{\partial \bar{w}}{\partial z}\right) \\
\gamma_{x y}=\frac{\partial \bar{v}}{\partial x}+\frac{\partial \bar{u}}{\partial y}+\left(\frac{\partial \bar{v}}{\partial x} \frac{\partial \bar{v}}{\partial y}+\frac{\partial \bar{w}}{\partial x} \frac{\partial \bar{w}}{\partial y}\right) \\
\varepsilon_{y y}=\varepsilon_{z z}=\gamma_{y z}=0
\end{gathered}
$$

where it has been assumed that, for moderate displacements,

$$
\left(\frac{\partial \bar{u}}{\partial x}\right)^{2}<<\frac{\partial \bar{u}}{\partial x},\left(\frac{\partial \bar{u}}{\partial x}\right)\left(\frac{\partial \bar{u}}{\partial z}\right)<<\left(\frac{\partial \bar{u}}{\partial x}\right)+\left(\frac{\partial \bar{u}}{\partial z}\right),\left(\frac{\partial \bar{u}}{\partial x}\right)\left(\frac{\partial \bar{u}}{\partial y}\right)<<\left(\frac{\partial \bar{u}}{\partial x}\right)+\left(\frac{\partial \bar{u}}{\partial y}\right)
$$

Substituting the displacement components to the strain-displacement relations, the strain components can be written as

$$
\begin{gathered}
\varepsilon_{x x}(x, y, z, t)=u^{\prime}+z \theta_{y}{ }^{\prime}-y \theta_{z}{ }^{\prime}+\frac{1}{2}\left(v^{\prime 2}+w^{\prime 2}\right) \\
\gamma_{x y}=v^{\prime}-\theta_{z} \quad \gamma_{x z}=w^{\prime}+\theta_{y}
\end{gathered}
$$

where $\gamma_{x y}, \gamma_{x z}$ are the additional angles of rotation of the cross-section due to shear deformation.

Considering strains to be small, employing the second Piola-Kirchhoff stress tensor and assuming an isotropic and homogeneous material, the stress components are defined in terms of the displacement ones as

$$
\begin{gathered}
S_{x x}=E\left[u^{\prime}+z \theta_{y}^{\prime}-y \theta_{z}^{\prime}+\frac{1}{2}\left(v^{\prime 2}+w^{\prime 2}\right)\right] \\
S_{x y}=G\left(v^{\prime}-\theta_{z}\right) \quad S_{x z}=G\left(w^{\prime}+\theta_{y}\right)
\end{gathered}
$$


On the basis of Hamilton's principle, the variation of the Lagrangian equation

$$
\delta \int_{t_{1}}^{t_{2}}\left(U-K-W_{e x t}\right) \mathrm{d} t=0
$$

expressed as a function of the stress resultants acting on the cross section of the column-pile in the deformed state, provide the governing equations and the boundary conditions of the column-pile subjected to nonlinear vibrations. In eqn (6), $\delta$ denotes variation of quantities while $U, K$ and $W_{\text {ext }}$ are the strain energy, the kinetic energy and the work by external load, respectively. Moreover, the stress resultants of the column-pile using the expressions of the stress components are given by

$$
\begin{aligned}
& N=E A\left[u^{\prime}+\frac{1}{2}\left(v^{\prime 2}+w^{\prime 2}\right)\right] \\
& M_{y}=E I_{y} \theta_{y}^{\prime} \quad M_{z}=E I_{z} \theta_{z}^{\prime} \\
& Q_{y}=G A_{y} \gamma_{x y} \quad Q_{z}=G A_{z} \gamma_{x z}
\end{aligned}
$$

where $A$ is the cross section area, $I_{y}, I_{z}$ the moments of inertia with respect to the principal bending axes and $G A_{y}, G A_{z}$ are its shear rigidities of the Timoshenko's beam theory, where

$$
A_{z}=\kappa_{z} A=\frac{1}{a_{z}} A \quad A_{y}=\kappa_{y} A=\frac{1}{a_{y}} A
$$

are the shear areas with respect to $y, z$ axes, respectively with $\kappa_{y}, \kappa_{z}$ the shear correction factors and $a_{y}, a_{z}$ the shear deformation coefficients. Substituting the stress components and the strain resultants to the strain energy variation and employing eqn (6), the equilibrium equations of the column-pile are derived as

$$
\begin{gathered}
-E A\left(u^{\prime \prime}+w^{\prime} w^{\prime \prime}+v^{\prime} v^{\prime \prime}\right)+\rho A \ddot{u}=p_{x} \\
E I_{z} v^{\prime \prime \prime \prime}+\rho A \ddot{v}+p_{s y}+\frac{E I_{z}}{G A_{y}}\left(\left(N v^{\prime}\right)^{\prime \prime \prime}-\rho A \frac{\partial^{2} \ddot{v}}{\partial x^{2}}-p_{s y}{ }^{\prime \prime}+p_{y}{ }^{\prime \prime}\right)-\left(N v^{\prime}\right)^{\prime} \\
-\rho I_{z} \frac{\partial^{2} \ddot{v}}{\partial x^{2}}-\frac{\rho I_{z}}{G A_{y}}\left(\frac{\partial^{2}\left(N v^{\prime}\right)^{\prime}}{\partial t^{2}}-\rho A \dddot{v}-\ddot{p}_{s y}+\ddot{p}_{y}\right)=p_{y}-m_{z}{ }^{\prime}
\end{gathered}
$$




$$
\begin{aligned}
E I_{y} w^{\prime \prime \prime}+\rho A \ddot{w}+p_{S z}+\frac{E I_{y}}{G A_{z}}\left(\left(N w^{\prime}\right)^{\prime \prime \prime}-\rho A \frac{\partial^{2} \ddot{w}}{\partial x^{2}}-p_{S z}{ }^{\prime \prime}+p_{z}{ }^{\prime \prime}\right)-\left(N w^{\prime}\right)^{\prime} \\
-\rho I_{z} \frac{\partial^{2} \ddot{w}}{\partial x^{2}}-\frac{\rho I_{y}}{G A_{z}}\left(\frac{\partial^{2}\left(N w^{\prime}\right)^{\prime}}{\partial t^{2}}-\rho A \dddot{w}-\ddot{p}_{s z}+\ddot{p}_{z}\right)=p_{z}+m_{y}^{\prime}
\end{aligned}
$$

Eqns (9) constitute the governing differential equations of a TimoshenkoRayleigh pile, partially embedded in viscoelastic foundation, subjected to nonlinear vibrations due to the combined action of time dependent axial and transverse loading. These equations are also subjected to the pertinent boundary conditions of the problem, which are given by

$$
\begin{gathered}
\alpha_{1} u(x, t)+\alpha_{2} N(x, t)=\alpha_{3} \\
\beta_{1} v(x, t)+\beta_{2} V_{y}(x, t)=\beta_{3} \quad \bar{\beta}_{1} \theta_{z}(x, t)+\bar{\beta}_{2} M_{z}(x, t)=\bar{\beta}_{3} \\
\gamma_{1} w(x, t)+\gamma_{2} V_{z}(x, t)=\gamma_{3} \quad \bar{\gamma}_{1} \theta_{y}(x, t)+\bar{\gamma}_{2} M_{y}(x, t)=\bar{\gamma}_{3}
\end{gathered}
$$

at the column-pile ends $x=0, l$, together with the initial conditions

$$
\begin{array}{cc}
u(x, 0)=\bar{u}_{0}(x) & \dot{u}(x, 0)=\dot{\bar{u}}_{0}(x) \\
v(x, 0)=\bar{v}_{0}(x) & \dot{v}(x, 0)=\dot{\bar{v}}_{0}(x) \\
w(x, 0)=\bar{w}_{0}(x) & \dot{w}(x, 0)=\dot{\bar{w}}_{0}(x)
\end{array}
$$

where $\bar{u}_{0}(x), \bar{v}_{0}(x), \bar{w}_{0}(x), \dot{\bar{u}}_{0}(x), \dot{\bar{v}}_{0}(x)$ and $\dot{\bar{w}}_{0}(x)$ are prescribed functions. In eqns (10b-e), $V_{y}, V_{z}, M_{y}, M_{z}$ and $\theta_{y}, \theta_{z}$ are the reactions, the bending moments and the angles of rotation due to bending with respect to $y, z$, respectively.

Finally, $\alpha_{k}, \beta_{k}, \bar{\beta}_{k}, \gamma_{k}, \bar{\gamma}_{k}(k=1,2,3)$ are functions specified at the columnpile ends $x=0, l$. Eqns (10) describe the most general nonlinear boundary conditions associated with the problem at hand and can include elastic support or restraint. It is apparent that all types of the conventional boundary conditions (clamped, simply supported, free or guided edge) can be derived from these equations by specifying appropriately these functions (e.g. for a clamped edge, $\alpha_{1}=\beta_{1}=\gamma_{1}=1, \bar{\beta}_{1}=\bar{\gamma}_{1}=1$ and all the other functions vanish).

The solution of the initial boundary value problem defined by eqns (9), subjected to the boundary conditions (10) and the initial conditions (11), and governing the nonlinear flexural dynamic of a Timoshenko-Rayleigh pile, partially embedded in viscoelastic foundation, requires the evaluation of the shear 
deformation coefficients $a_{y}, a_{z}$, corresponding to the principal coordinate system $C y z$. These coefficients are established by equating the approximate formula of the shear strain energy per unit length [15]

$$
U_{\text {appr. }}=\frac{a_{y} Q_{y}^{2}}{2 A G}+\frac{a_{z} Q_{z}^{2}}{2 A G}
$$

with the exact one given by

$$
U_{\text {exact }}=\int_{\Omega} \frac{\left(\tau_{x z}\right)^{2}+\left(\tau_{x y}\right)^{2}}{2 G} \mathrm{~d} \Omega
$$

and are obtained as [16]

$$
\begin{aligned}
& a_{y}=\frac{1}{\kappa_{y}}=\frac{A}{\Delta^{2}} \int_{\Omega}[(\nabla \Theta)-\boldsymbol{e}] \cdot[(\nabla \Theta)-\boldsymbol{e}] \mathrm{d} \Omega \\
& a_{z}=\frac{1}{\kappa_{z}}=\frac{A}{\Delta^{2}} \int_{\Omega}[(\nabla \Phi)-\boldsymbol{d}] \cdot[(\nabla \Phi)-\boldsymbol{d}] \mathrm{d} \Omega
\end{aligned}
$$

where $\left(\tau_{x z}\right)_{j},\left(\tau_{x y}\right)_{j}$ are the transverse (direct) shear stress components,

$$
\nabla \equiv \boldsymbol{i}_{\boldsymbol{y}}(\partial / \partial y)+\boldsymbol{i}_{\boldsymbol{z}}(\partial / \partial z)
$$

is a symbolic vector with $\boldsymbol{i}_{\boldsymbol{y}}, \boldsymbol{i}_{z}$ the unit vectors along $y$ and $z$ axes, respectively. Moreover,

$$
\Delta=2(1+v) I_{y} I_{z}
$$

where $v$ is the Poisson ratio of the cross section material,

$$
\boldsymbol{e}=\left(v I_{y} \frac{y^{2}-z^{2}}{2}\right) \boldsymbol{i}_{\boldsymbol{y}}+v I_{y} y z \boldsymbol{i}_{z} \text { and } \boldsymbol{d}=v I_{z} y z \boldsymbol{i}_{\boldsymbol{y}}-\left(v I_{z} \frac{y^{2}-z^{2}}{2}\right) \boldsymbol{i}_{z}
$$

while $\Theta(y, z)$ and $\Phi(y, z)$ are stress functions which are evaluated from the solution of the following Neumann type boundary value problems [16]

$$
\nabla^{2} \Theta=-2 I_{y} y \quad \text { in } \Omega \quad \text { and } \quad \frac{\partial \Theta}{\partial n}=\boldsymbol{n} \cdot \boldsymbol{e} \text { on } \Gamma=\bigcup_{j=1}^{K+1} \Gamma_{j}
$$




$$
\nabla^{2} \Phi=-2 I_{z} z \quad \text { in } \Omega \quad \text { and } \quad \frac{\partial \Phi}{\partial n}=\boldsymbol{n} \cdot \boldsymbol{d} \text { on } \Gamma=\bigcup_{j=1}^{K+1} \Gamma_{j}
$$

where $\boldsymbol{n}$ is the outward normal vector to the boundary $\Gamma$. In the case of negligible shear deformations $a_{z}=a_{y}=0$. It is also worth noting here that the boundary conditions (13b), (14b) have been derived from the physical consideration that the traction vector in the direction of the normal vector $\boldsymbol{n}$ vanishes on the free surface of the column-pile.

\section{Integral representations: numerical solution}

According to the preceding analysis, the nonlinear flexural dynamic analysis of a Timoshenko-Rayleigh pile, partially embedded in viscoelastic foundation, undergoing moderately large deflections reduces to determining the displacement components $u(x, t)$ and $v(x, t), w(x, t)$, having continuous derivatives up to the second order and up to the fourth order with respect to $x$, respectively, and also up to the second order with respect to $t$ (ignoring the inertia terms of the fourth order [17]). These displacement components must satisfy the coupled governing differential eqns (9) inside the column-pile, the boundary conditions (10) at the column-pile ends $x=0, l$ and the initial conditions (11). Eqns (9) are solved using the Analog Equation Method [10] as developed for solving hyperbolic differential equations [18].

\section{Numerical examples}

On the basis of the presented analytical and numerical procedures, a computer program has been written and representative examples have been studied to demonstrate the efficiency of the developed method.

\subsection{Example 1}

A column-pile monolithically connected to a bridge deck is embedded in two layers of cohesive soil and excited by seismic motions. The concentrated mass at the centre of the deck is $60 \mathrm{t}$, the height of the column is $10 \mathrm{~m}$, the embedment length of the pile is $30 \mathrm{~m}$ and the diameter of the column-pile equals $1.5 \mathrm{~m}$. The material of the column-pile is assumed to be linear elastic. The layered soil profile is characterized by a soft to medium normally consolidated clay set on top of a stiff clay, while the rigid bedrock is encountered at $-50 \mathrm{~m}$. The soft clay has a thickness of $18 \mathrm{~m}$ and the undrained shear strength is assumed to follow a linear law, i.e. $S_{u}=2 z(\mathrm{kPa})$, where $z$ is the depth. The second layer is $32 \mathrm{~m}$ thick and has constant undrained shear strength of $100 \mathrm{kPa}$. 
The influence of shaking on the seismic response is investigated by selecting two well-known acceleration records as seismic excitations:

- the record from Aegion earthquake (1995)

- the record from Lefkada earthquake (2003)

These records were chosen as two strong motions of the seismic environment of Greece, with one and many cycles, respectively. The records were first scaled to a Peak Ground Acceleration (PGA) of $0.8 \mathrm{~g}$ at the ground surface. Then, through one dimensional shear wave propagation analysis, the bedrock motions as well as the motions at various depths along the pile were estimated.

Figs. 1 and 2 show, respectively, the acceleration and displacement time histories, corresponding to Lefkada excitation motion, at the bridge deck level and at the ground surface. The results obtained from both the geometrically linear and nonlinear analysis, taking into account both rotary inertia and the shear deformation effect are depicted, while the maximum values of divergence between the two approaches is also presented. Similarly, figs. 3 and 4 show, respectively, the acceleration and displacement time histories, corresponding to Aegion excitation motion at the bridge deck level and at the ground surface.

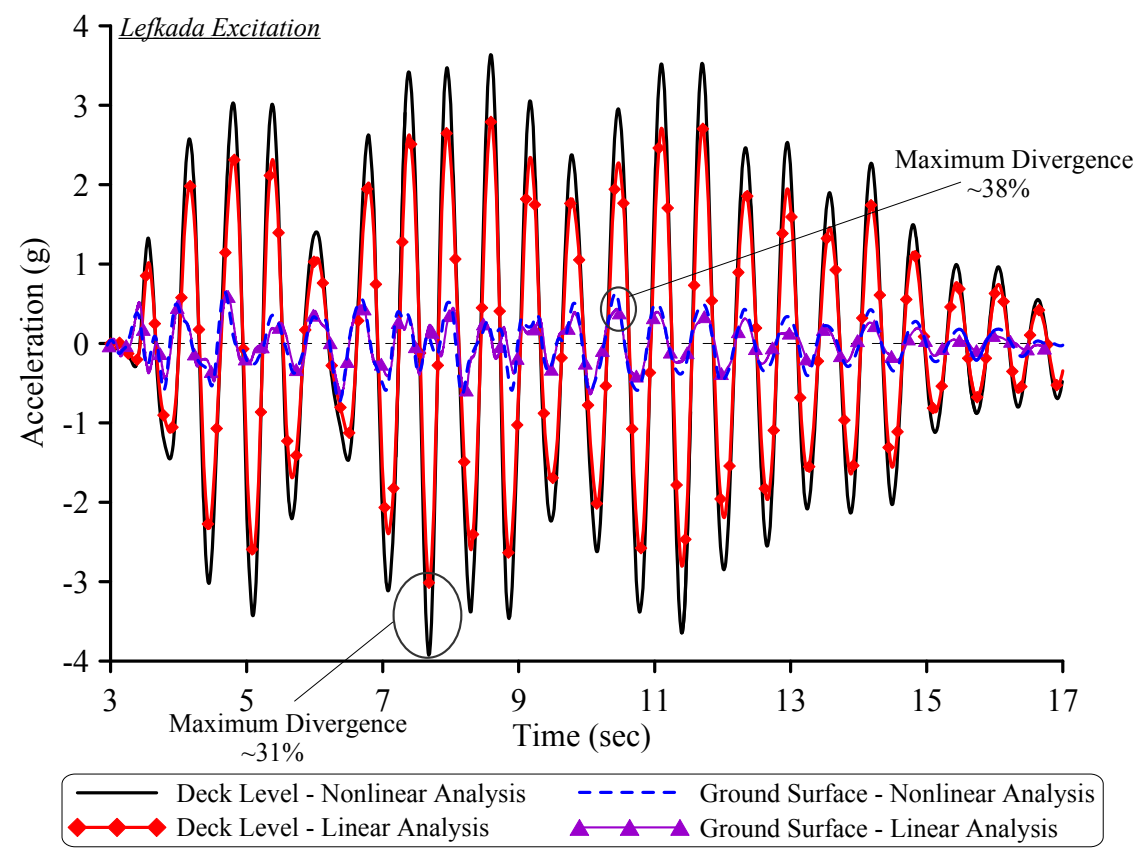

Figure 1: Acceleration time history of the deck level and ground surface for Lefkada excitation. 


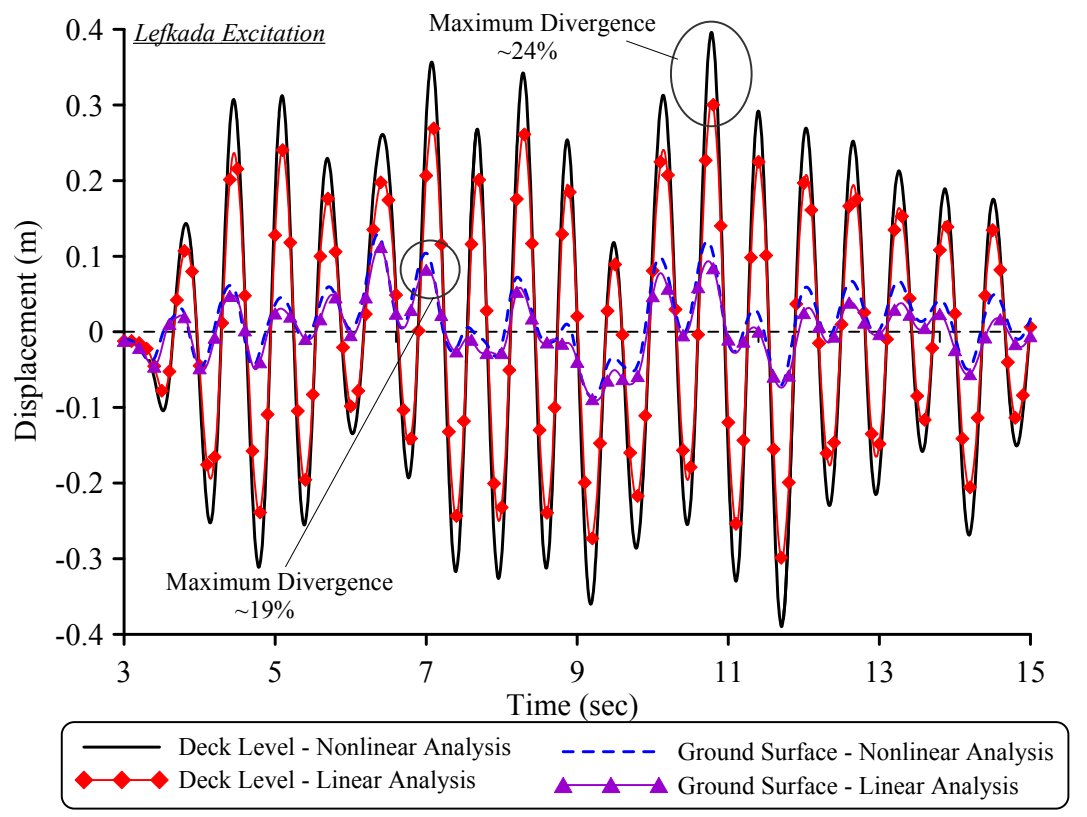

Figure 2: Displacement time history of the deck level and ground surface for Lefkada excitation.

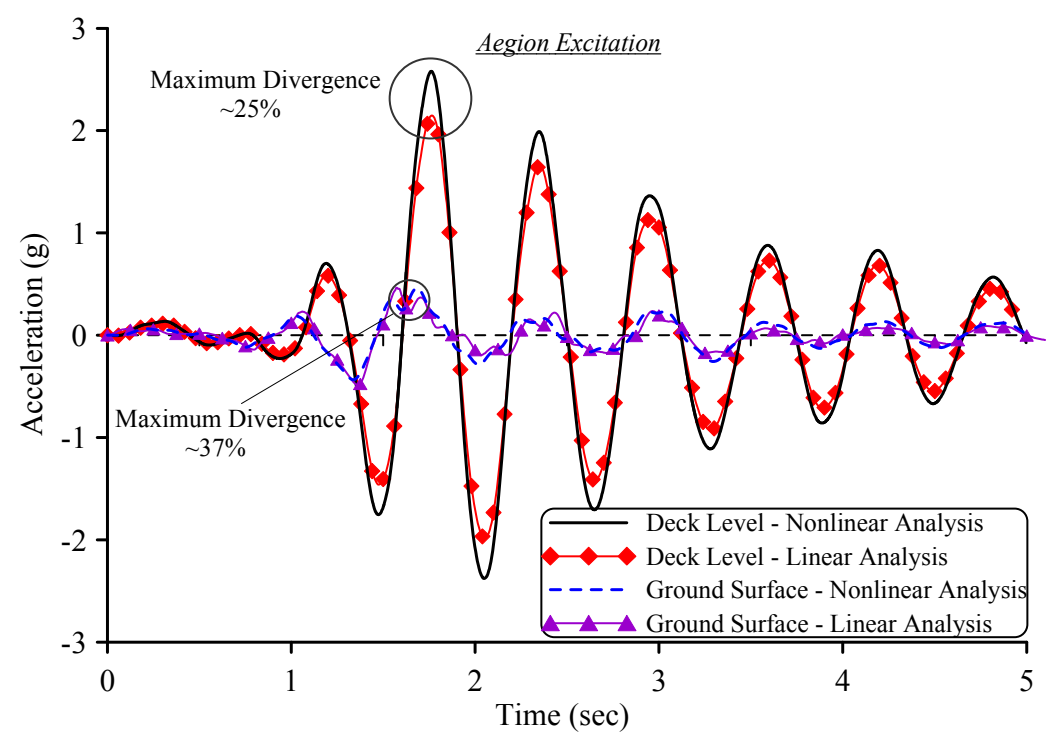

Figure 3: Acceleration time history of the deck level and ground surface for Aegion excitation. 


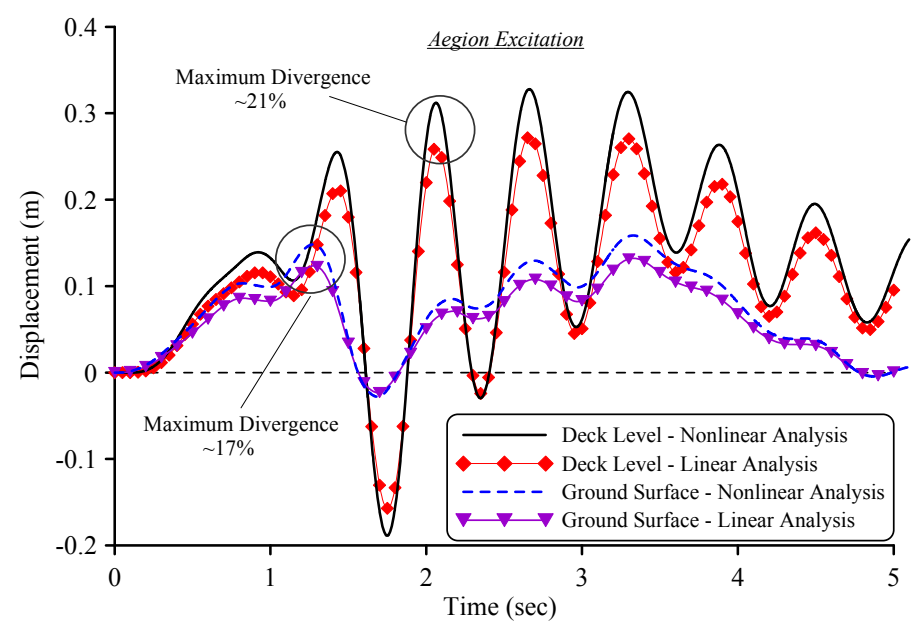

Figure 4: Displacement time history of the deck level and ground surface for Lefkada excitation.

Finally, in fig. 5, the maximum bending moment distributions are presented for the aforementioned seismic excitations, performing either linear or nonlinear analysis. From the obtained results, it is observed that the discrepancy between the results of linear and nonlinear analysis is of great importance and cannot be ignored, especially in cases of bridge column-piles where the applied axial load is of high magnitude.

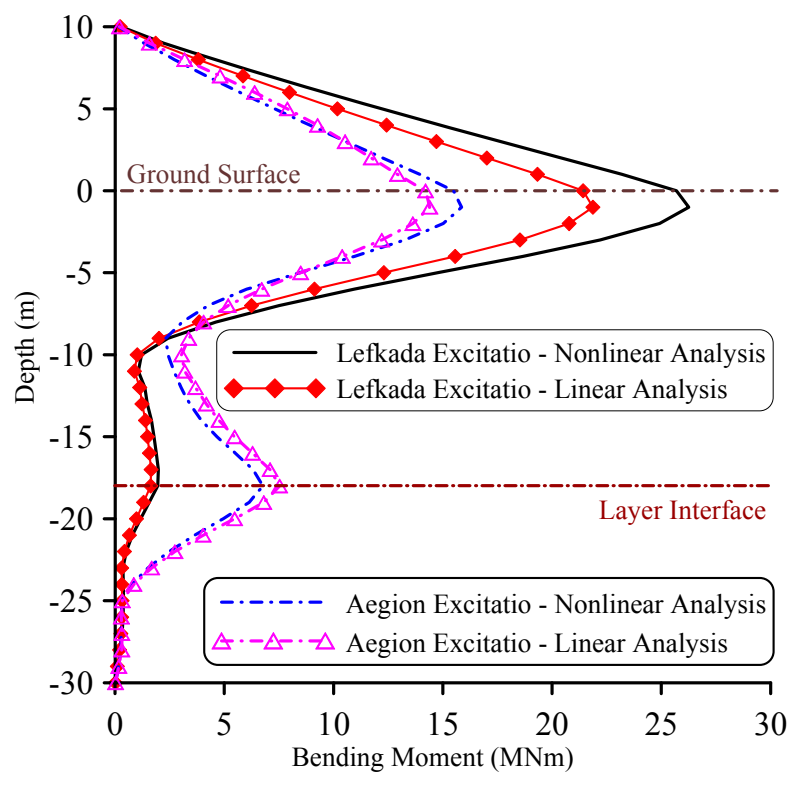

Figure 5: Bending moment envelopes for Lefkada and Aegion excitations. 


\subsection{Example 2}

As a second example, a partially embedded column-pile of a total length of $10 \mathrm{~m}$ $\left(l_{\text {free }}=3.0 \mathrm{~m}, l_{\text {embed }}=7.0 \mathrm{~m}\right)$, of circular cross section with a diameter of $0.5 \mathrm{~m}$ is analysed. The foundation model is characterized by the Winkler modulus $k=17.4$ $\mathrm{kN} / \mathrm{m}^{2}$ and the damping coefficient $c$. Regarding its boundary conditions, the embedded pile end is considered free, while the other end is free to move but rotationally constrained. The column-pile is subjected to a concentrated compressive axial load $P_{x}(0, t)=1.5 \mathrm{MN},(t \geq 0)$ and to a concentrated transverse force $P_{z}(0, t)=1 \mathrm{MN},(t \geq 0)$ acting at its top.

In table 1 , the maximum values of the head displacement and the periods of the first-cycle of motion are presented taking into account the rotary inertia and the shear deformation effect, for two values of the damping coefficient, $\left(c=0 \mathrm{kNs} / \mathrm{m}^{2}\right.$, $\left.c=12 \mathrm{kNs} / \mathrm{m}^{2}\right)$ and performing either linear or nonlinear analyses.

Finally, in order to demonstrate the coupling effect of the transverse displacements in both directions in the nonlinear analysis, as a variant of the above application, the examined column-pile additionally to the already described loading is also subjected to a concentrated transverse force $P_{y}(0, t)=2 \mathrm{MN}$, acting also at its top. In table 2, the maximum values of the head transverse displacements are presented performing either linear or nonlinear analyses. The difference in the elements of the first columns of tables 1 and 2 is due to the coupling effect of the transverse displacements.

Table 1: $\quad$ Maximum pile head displacement $w_{\text {top }}^{\max }(\mathrm{cm})$ and period $T_{z} \times 10^{-2}(\mathrm{~s})$ of the first cycle of motion.

\begin{tabular}{|c|c|c|c|}
\hline \multicolumn{4}{|c|}{ Undamped Case } \\
\hline Nonlinear Analysis & \multicolumn{2}{c|}{ Linear Analysis } \\
\hline$w_{\text {top }}^{\max }$ & $T_{z}$ & $w_{\text {top }}^{\max }$ & $T_{z}$ \\
\hline 29.66 & 8.10 & 26.96 & 7.28 \\
\hline \multicolumn{3}{|c|}{ Damped Case $c=12 \mathrm{kNs} / \mathrm{m}^{2}$} \\
\hline Nonlinear Analysis & \multicolumn{2}{c|}{ Linear Analysis } \\
\hline$\left(w_{\text {ton }}\right)$ & $T_{z}$ & $\left(w_{\text {ton }}\right)$ & $T_{z}$ \\
\hline 28.97 & 7.78 & 26.30 & 7.36 \\
\hline
\end{tabular}

Table 2: Maximum pile head transverse displacements $w_{\text {top }}^{\max }, v_{\text {top }}^{\max }(\mathrm{cm})$.

\begin{tabular}{|c|c|c|c|}
\hline \multicolumn{2}{|c|}{ Nonlinear Analysis } & \multicolumn{2}{c|}{ Linear Analysis } \\
\hline$w_{t o D}^{\max }$ & $v_{\text {top }}^{\max }$ & $w_{\text {top }}^{\max }$ & $v_{\text {top }}^{\max }$ \\
\hline 29.67 & 59.343 & 26.97 & 53.912 \\
\hline
\end{tabular}




\section{Concluding remarks}

The main conclusions that can be drawn from this investigation are:

a. The numerical technique presented in this investigation is well suited for computer aided analysis for column-piles of arbitrary simply or multiply connected doubly symmetric cross section.

b. The proposed model takes into account both kinematic and inertial interaction in the geometrical nonlinear dynamic response of a column-pile embedded in a layered soil profile.

c. The discrepancy between the results of the geometrically linear and the nonlinear analyses is significant.

d. The damping coefficient is of paramount importance for piles in viscoelastic foundations, as it reduces the vibration amplitude and the consequences of the dynamic response.

e. The shear deformation increases the transverse displacements and decreases the bending moments in both linear and nonlinear analysis.

\section{Acknowledgement}

The work of this paper was conducted from the "DARE" project, financially supported by a European Research Council (ERC) Advanced Grant under the "Ideas" Programme in Support of Frontier Research [Grant Agreement 228254].

\section{References}

[1] Hetenyi, M., Beams and plates on elastic foundations and related problems. Applied Mechanics Reviews, 19, pp. 95-102, 1966.

[2] Rades, M., Dynamic analysis of an inertial foundation model. International Journal of Solids and Structures, 8, pp. 1353-1372, 1972.

[3] Wang, T.M. \& Stephens, J. E., Natural frequencies of Timoshenko beams on Pasternak foundation. Journal of Sound and Vibration, 51(2), pp. 149-155, 1977.

[4] De Rosa, M.A., Free vibrations of Timoshenko beams on two-parameter elastic foundation. Computers \& Structures, 57(1), pp. 151-156, 1995.

[5] El-Mously, M., Fundamental frequencies of Timoshenko beams mounted on Pasternak foundation. Journal of Sound and Vibration, 228(2), pp. 452-457, 1999.

[6] El Naggar, M.H. \& Novak, M., Nonlinear analysis for dynamic lateral pile response. Soil Dynamics and Earthquake Engineering, 15, pp. 233-244, 1996.

[7] Pardon, L.A., Aznarez, J.J. \& Maeso, O., BEM-FEM coupling model for the dynamic analysis of piles and pile groups. Engineering Analysis with Boundary Elements, 31, pp. 473-484, 2007.

[8] Hu, C.L., Cheng, C.J. \&Chen, Z.X., Nonlinear transverse free vibrations of piles. Journal of Sound and Vibration, 317, pp. 937-954, 2008. 
[9] Lewandowski, R., Nonlinear free vibrations of multispan beams on elastic supports. Computers \& Structures, 32(2), pp. 305-312, 1989.

[10] Katsikadelis, J.T., The analog equation method. A boundary-only integral equation method for nonlinear static and dynamic problems in general bodies. Theoretical and Applied Mechanics, 27, pp. 13-38, 2002.

[11] Chang, S. Y., Studies of Newmark method for solving nonlinear systems: (I) basic analysis. Journal of the Chinese Institute of Engineers, 27(5), pp. 651-662, 2004.

[12] Isaacson, E. \& Keller, H.B., Analysis of Numerical Methods, John Wiley and Sons: New York, 1966.

[13] Timoshenko, S.P. \& Goodier, J.N., Theory of Elasticity, 3rd ed., McGrawHill: New York, 1984.

[14] Cowper, G.R., The shear coefficient in Timoshenko's beam theory. Journal of Applied Mechanics, ASME, 33(2), pp. 335-340, 1966.

[15] Stephen, N.G., Timoshenko's shear coefficient from a beam subjected to gravity loading. ASME Journal of Applied Mechanics, 47, pp. 121-127, 1980.

[16] Sapountzakis, E.J. \& Mokos, V.G., A BEM solution to transverse shear loading of beams. Computational Mechanics, 36, pp. 384-397, 2005.

[17] Thomson, W. T., Theory of vibration with applications, Prentice Hall: Englewood Cliffs, 1981.

[18] Sapountzakis, E.J. \& Katsikadelis, J.T., Elastic deformation of ribbed plates under static, transverse and inplane loading. Computers and Structures, 74, pp. 571-581, 2000. 